\title{
Usability of "Traysi": A Web Application for Tricycle Commuters
}

\author{
Ertie C. Abana \\ School of Engineering, Architecture \\ Interior Design and Information Technology Education \\ University of Saint Louis, Tuguegarao City \\ Cagayan, Philippines
}

\begin{abstract}
This study measured the usability of a web application for tricycle commuters that was developed using Hypertext Markup Language (HTML), Cascading Style Sheet (CSS) and Javascript (JS) with the aid of Google Artificial Programming Interfaces (APIs). Toward this goal, the effectiveness, efficiency and user satisfaction were measured using common usability metrics. The effectiveness was measured in terms of task completion rate and user errors while efficiency was measured in terms of time on task. For user satisfaction, the post-task questionnaire, Single Ease Question (SEQ), was used. In order to check whether the web application will be usable even for first time users, the usability test was conducted three times. The result revealed that the usability of the web application is acceptable on the first trial. However, the usability improved on the next used which is evident on the third trial that yielded a 93.33\% task completion rate with only one user error. The average time on task on every trial was lower than the maximum acceptable task time and the user satisfaction was high $(\overline{\mathbf{x}}=\mathbf{6 . 0 0})$. Thus, the web application was highly usable in doing its intended purpose especially if it is repeatedly used.
\end{abstract}

Keywords-Tricycle; usability metrics; web application; fare calculation; Google API

\section{INTRODUCTION}

In most developing nations, motorcycles are one of the primary modes of transportation. In the Philippines, local public transport in the form of tricycles exists [1]. The design differs from one town to another [2] but is mostly made of a motorcycle fitted with a sheet-metal body frame covered by roof canvas [3] to accommodate local travelers. Tricycles provide services by transporting a commuter [4] to major streets and barangays with low fuel consumption [5]. The tricycle is notable for their role as gap fillers [6]. They exist mostly to fill service voids left by busses and other formal public transport vehicles.

Tuguegarao City, a developing town in the Philippines considered to be the heart of Region 2 where the Regional Offices are located, still uses the tricycle as the primary mode of transportation. It was first introduced in the early 1960s and by 2010 it has reached a shocking number of 12,000 tricycles going around the city. Unfortunately, Tuguegarao has been branded as the city of English-speaking tricycle drivers because of the recorded cases of abuse by drivers. Some tricycle drivers in the city collect unjust fares from the passengers [2] that present a negative image to Tuguegarao. Although the local government has been working hard to apprehend these abusive drivers, there are still some that are overcharging. Most of the time, those people who are new to the place fall victims to this wrong practice by the abusive drivers. The government has imposed the posting of fare rates inside the tricycle fronting the passenger [2] but drivers who charge more than the prescribed fare rates still exists. It seems that the only way to stop this practice is to educate the passengers regarding fare rates. Moreover, the use of automated fare systems to tricycles is still farfetched since this will be viewed as additional expenses to both drivers and passengers.

In educating passengers about the prescribed fare rates, researchers looked at the possibility of using technology. A real-time trip information service was developed in Singapore for passengers to determine the estimated duration and fare of a trip that they will take so that they can plan their time and budget accordingly as well as serving as a safeguard against abusive drivers who might take longer than expected routes especially when servicing tourists [7]. In the Philippines, a web application called Sakay.ph [8] is now used by many passengers in Metro Manila to compute the fares for the given origin and destination point.

Most of the existing studies that developed an application for commuters only incorporates buses, trains, and jeepneys in the travel guide. Although there are some applications for tricycle commuters developed for specific places, none have studied the usability of such applications to better understand how real users interact with the apps which can serve as a guide to improve the application based on the results. This study attempted to address this gap. A web application called "Traysi" was developed and its usability in terms of effectiveness, efficiency and satisfaction was evaluated to determine if it can provide the tricycle commuters a usable application to query the fare and at the same time get the travel direction and tricycle terminal location address when going to a certain place.

The design and development of the web application were done using HTML, CSS, JS and Google APIs. For the usability testing, a timekeeper and an observer were involved to get an accurate result. The results of the usability testing can serve as a guide for developers to improve existing applications. Future developers will also be benefited as they will have an idea on to what aspect of the design, they will focus more on their own version of the application for tricycle commuters. 


\section{Methodology}

\section{A. Description of the Web Application Evaluated}

The web application "Traysi" is a tricycle fare calculation web application that uses the latest fare matrix approved in Tuguegarao City. The minimum regular fare imposed by the city government was set to be Php12.00 that corresponds to three (3) kilometers. The distance covered from origin to destination in each individual trip is different, so to address this, the city government approved the petition of the Tuguegarao City Tricycle Operators and Drivers Association (TCTODA) wherein a $\mathrm{P} 0.50$ per kilometer of the succeeding kilometers (after the three (3) kilometers) will be added to the fare.

The web application was developed using HTML, CSS and JS. HTML is a typical markup language used in developing the web application. With CSS and JS, the functionality and interface design of the web application was formulated. Moreover, several Google Maps subroutine definitions called API were utilized in the development of the web application. The APIs include Maps Embed API, Distance Matrix API, Directions API, and Places API. The web application is powered by 000webhost, a free web hosting site.

The pinning of origin and destination were facilitated by Google Map's autocomplete place handler as shown in Fig. 1a. This handler speeds up the interaction between the web app and the user. It predicts the place a user intends to enter after a few characters have been typed into the origin and destination text field. After the origin and destination have been identified, the web application displayed the actual direction on the embedded Google Map as shown in Fig. 1-b. The calculated fare is revealed when the user taps the Calculate button. Also, the tricycle terminal location address of the barangay where the user wants to go was shown at the bottom of the web application as shown in Fig. 1-c.

\section{B. Usability Testing}

Several systems are evaluated to determine if its suites the need of the users. The evaluation of a system is oftentimes done by measuring its usability. The goals that a particular study wants to achieve are where the choice of measures depends [9]. The definition of usability from ISO 9241-11 is "the extent to which a product can be used by specified users to achieve specified goals with effectiveness, efficiency, and satisfaction in a specified context of use" [10]. Usability is commonly measured by the three metrics: effectiveness, efficiency, and satisfaction [9][11][12][13]. The usability of the web application named "Traysi" was determined and analyzed using these three metrics.

Effectiveness is defined in the ISO9241-11 as "the accuracy and completeness with which users achieve specified goals". This usability metric only takes into account the extent to which a goal was achieved, not on how it was achieved. It can be calculated by measuring the task completion rate [9][11][12][13]. The task completion rate is considered as a fundamental usability metric. It can be determined by assigning a binary value of ' 1 ' for every achieved task and ' 0 ' for the unachieved task by a participant [11][13]. In the study, task completion was calculated as a percentage [13] using (1) wherein NTCS is the number of tasks completed successfully and TNTU is the total number of tasks undertaken.

$$
\text { Task Completion Rate }=(\text { NTCS } / T N T U) \times 100 \%
$$

Another measurement of effectiveness involves counting the total errors [9][11][12] a participant makes when completing a task. This is important because it provides diagnostic information [12] on the system being evaluated. The errors can be of many forms like mistakes, unintended actions, slips or omissions that a participant makes while performing a task.

Efficiency is another important usability metric that refers to the resources spent in achieving a task [14]. It can be measured in terms of the mean time taken to achieve a specific task [9][13]. The most common measure of efficiency in the evaluation of usability is time on task [9][15]. A stopwatch was used in getting the time on task for every user. The average task time for every trial was estimated using the geometric mean. The geometric mean was used since the study has a small sample size. It was recommended by a recent study [16] for small sample sizes $(n<25)$ because it showed consistently less error and bias than the median or the trim-top mean.

Satisfaction is the subjective response of the user after a usability test [9][13]. It can be measured through standardized questionnaires that are built using a Likert scale [9]. A variety of questionnaires can be used to measure user satisfaction but the cheapest and quickest to administer [17] is Single Ease Questionnaire (SEQ). SEQ is a Likert scale variant that is found to be more reliable [18] in small sample sizes. This task level satisfaction metric was given after the users attempted the task regardless if the task was completed or not. The SEQ is composed of only one question that has seven levels as shown in Fig. 2.

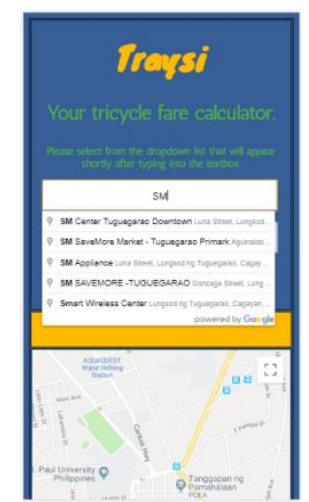

(a)

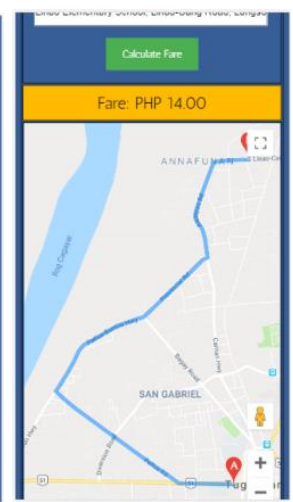

(b)

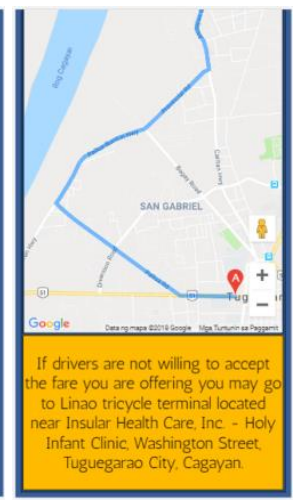

(c)
Fig. 1. (a) Autocomplete Place, (b) Tricycle Direction and Fare, (c) Tricycle Terminal Location Address.

\section{Overall, the task was?}

$\begin{array}{lllllll}\text { Very } & & & & & & \text { Very } \\ \text { Difficult } & & & & & \text { Easy } \\ 1 & 2 & 3 & 4 & 5 & 6 & 7\end{array}$

Fig. 2. The Single Ease Question (SEQ). 


\section{Participants of the Study}

The research participants of the study consisted of 15 individuals that were conveniently selected to use the web application using their own mobile phones that have an internet connection. These participants are regular tricycle passengers in Tuguegarao City.

\section{Data Gathering Tools}

For the usability data, a timekeeper used a stopwatch in recording the time spent by a participant in using the web application. Meanwhile, an observer was tasked to record the errors committed by the participant. This observer also determined if the task is completed or not. The usability data for each trial were all recorded in a Microsoft Excel workbook.

\section{E. Data Gathering Procedure}

The data gathering in the study was done separately per participant. The usability measures were collected through experimental methods. Every participant was informed that they may not complete the task and may close or reload the web application anytime they want to. The participant was given the task to use the application by inputting their preferred origin and destination on the web application. The time spent doing the task was collected by a time-keeper. Meanwhile, the observer recorded the number of errors the participant committed while doing the task. The participant would inform the observer upon completion of the task. After doing the task, the participants were asked about the satisfaction in doing the task. The usability testing was done three times to determine if the results changes on the next use.

\section{RESULTS AND DISCUSSION}

The study measured the usability of a web application of tricycle commuters named "Traysi". Usability was measured in terms of effectiveness, efficiency, and satisfaction. Reference [17] mentioned that simultaneously measuring these three dimensions provides the best quality data in usability studies.

\section{A. Effectiveness}

Fig. 3 describes the effectiveness of the web application based on task completion rate. On the first trial, 11 out of 15 participants were able to complete the task of calculating the fare. It had to be noted that these participants were first time users of the web application being studied. The task completion rate improved on the second trial wherein 12 of the participants were able to successfully complete the task. On the third trial, a significantly higher completion rate was computed because only one participant was not able to successfully complete the task.

Majority of the participants on the first trial were able to finish the task even though it was their first time using the web application. This is because the web application had short, succinct and self-describing text content to give participants the idea of what kind of data to enter in the textboxes. This is one of the desirable factors for a good user interface [19][20]. Additionally, the design of the web application is user-centered [21] wherein users can run the application without prior training. The second trial had a much higher completion rate but still, some of the participants were not able to complete the task despite completing it on the first trial. They were trying something out that resulted in an unsuccessful task. The third trial, however, had a very high task completion rate which is over the average task completion rate benchmark of $78 \%$ used in other studies [22][23].

Some errors were made by the participants when attempting to complete the task of calculating fare as shown in Fig. 4. The greatest number of errors were recorded on the first trial. This, however went down on the second and third trial. The user errors included not selecting a place from the dropdown list, selecting a place outside the town, no place selected in the origin nor destination, and accidentally going back to the previous page. Some of the participants committed errors on every trial. But the number of errors still went down on the next use. This showed that when users already had knowledge of using the application, there will only be a small chance of them committing the same error again. Moreover, user errors are inevitable and are often observed in human-computer interaction systems [24][25].

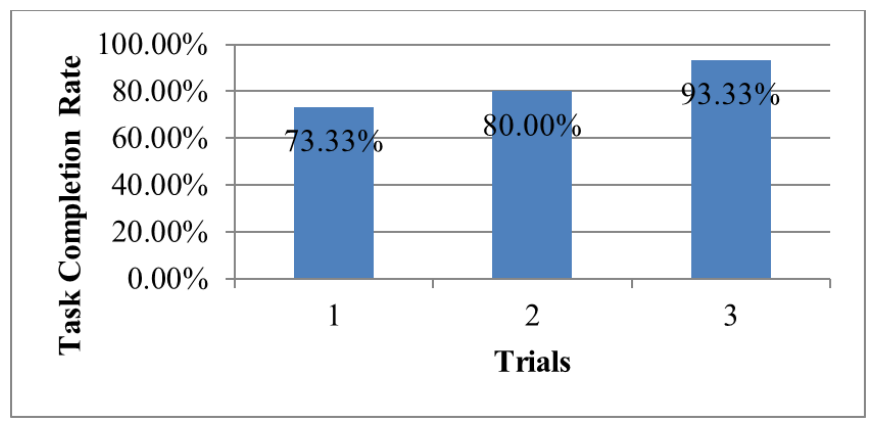

Fig. 3. Task Completion Rate from Each Trial.

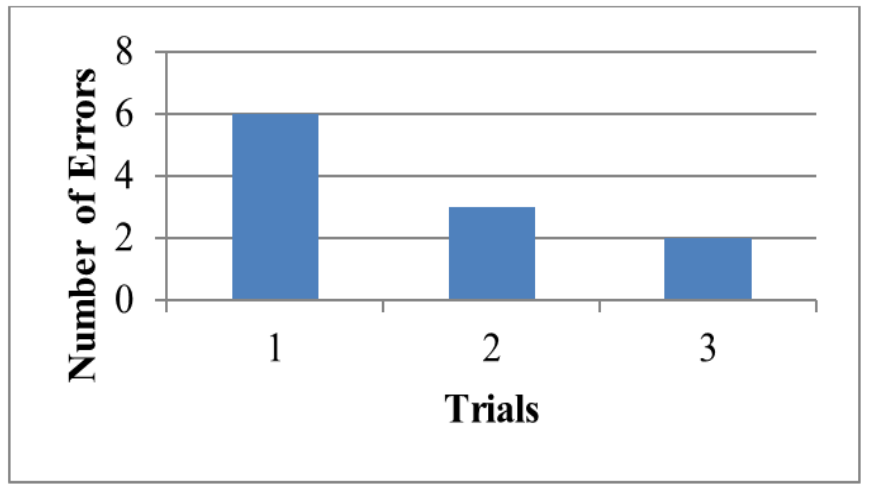

Fig. 4. Number of user Errors from Each Trial.

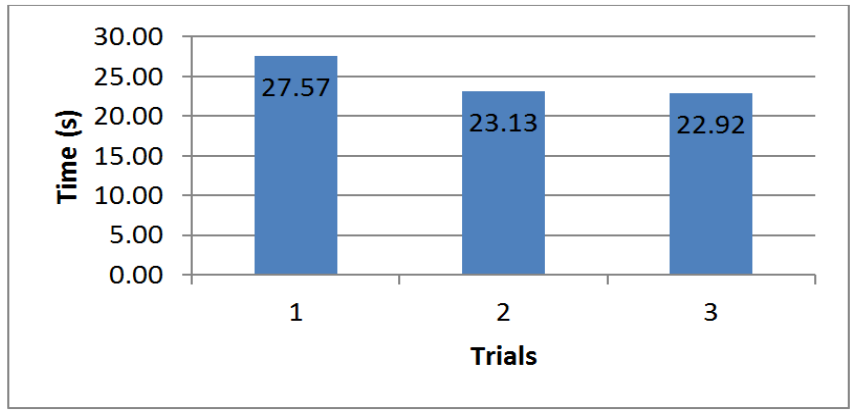

Fig. 5. Average Time on Task from Each Trial. 


\section{B. Efficiency}

The average time on task of the participants from each trial is presented in Fig. 5. The first trial yields the highest average time on task, nevertheless, it decreased on the succeeding trials. The difference between the second and third trial is very small. This was due to the slow internet connection that made the participants wait for the information to load even if they already knew how to use the web application.

The average time on task of the participants from all the trials were lower than the maximum acceptable task time which is 40.98 seconds, 34.58 seconds and 36.65 seconds, respectively. The maximum acceptable task time is computed using the method proposed by [24]. It is done by removing the times from failed tasks first then removing the times where satisfaction scores are less than 5.6 for 7 -point scale and finally getting the 95th percentile of the remaining times to arrive at the specification limit.

The positive result from the efficiency testing can be attributed to the card base design in the user interface wherein participants were only limited to one screen so that they may visualize the data well and not worry with where to go or what to click next. The implementation of card base design method bridged the gap between interaction and usability [19] in a synchronized manner to create a consistent user interface design across devices. The only problem though was the slow internet connection which makes the results to load longer than expected. This was also a problem with another internet-based system [14] because the Philippines is one of the countries with the slowest internet connections in Asia.

\section{Satisfaction}

Fig. 6 describes the satisfaction of users in using the web application particularly on doing the task of calculating tricycle fare. Only some of the participants were somewhat satisfied on the app in the first trial $(\overline{\mathrm{x}}=4.73)$ but eventually giving a high satisfaction on the second trial $(\overline{\mathrm{x}}=5.47)$ and third trial $(\overline{\mathrm{x}}=$ $6.00)$. Four participants perceived that the task was very easy after using the web application for the third time.

It was evident in the results that the user satisfaction was associated with task completion and errors. Those participants that were able to complete the task and did not make errors perceived that the task was very easy while those participants that were not able to complete the task and made errors perceived that the task was difficult. This is consistent with the study of [17] where the task completion rate and error correlate with satisfaction.

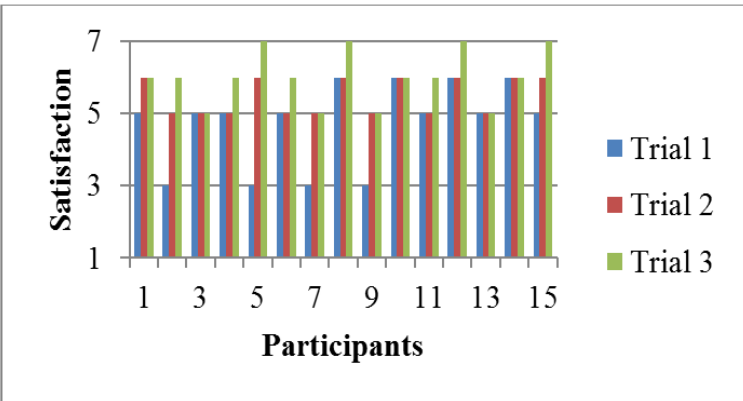

Fig. 6. User Satisfaction on the Ease of use of the Web Application.

\section{CONCLUSION}

This study determined if the web application for tricycle commuters named "Traysi" can provide the tricycle commuting public a usable application to query the fare and at the same time get the travel direction and tricycle terminal location address when going to a certain place by measuring its usability. It was only limited to the usability metrics that include effectiveness, efficiency, and satisfaction. Other software metrics were not used to evaluate the web application. The effectiveness of the web application in calculating the fare, displaying the travel direction and providing the tricycle terminal location address was evident based on the high task completion rate and low number of user errors even on the first used. Also, the web application was efficient in displaying the information on the screen despite slow internet connectivity. The participants also perceived that the task of using the application by inputting the preferred origin and destination is easy. Based on the findings presented, the web application named "Traysi" has a high level of usability. Moreover, this level of usability increases on the next use of the application.

For future works, a feature which will automatically show the tricycle terminal location in the map by clicking on the information provided can be added in the app. Additionally, a mobile application version can also be developed. In future usability testing, it should be done in a lab where all participants will do the task at the same time to get a more accurate result.

\section{ACKNOWLEDGMENT}

I am thankful to the University of Saint Louis for providing valuable help during the development of the application. I would like also to thank the staffs of the University Research and Development Center for technical guidance.

REFERENCES

[1] N. J. L. Dorado, P. D. C. Fabros, \& C. A. N. Rupisan, "An ergonomic analysis of tricycle sidecars in Quezon City," Procedia Manufacturing, 3, pp. 2816-2823, 2015.

[2] D. C. Agustin, N. C. Costales, M. Gadingan, L. M. Francisco, \& E. Fronda, "The functionality of tricycle regulation unit of Tuguegarao City," International Journal of Advanced Research in Management and Social Sciences, 7(3), pp. 211-236, 2018.

[3] O. O. Ipingbemi, \& A. Adebayo, "Tricycle as a mode of public transportation in Ibadan Metropolis, Nigeria," IFE Research Publications in Geography, 14(1), pp. 87-100, 2017.

[4] F. Balaria, "Cabanatuan City: Tricycle capital of the Philippines," International Journal of Management and Commerce Innovations, 4(1), pp. 307-314, 2016.

[5] D. Declan, "An empirical study of the use of tricycle as a public transport mode in Nigerian cities," Journal of Social Sciences and Public Affairs, 2(20), pp. 66-76, 2012.

[6] O. R. Nwaogbe, C. C. Ibe, \& S. I. Ukaegbu, "Quality of the paratransit service (tricycle) and its operation in Aba, Nigeria: An analysis of customers' opinions," Journal of Transport and Supply Chain Management, 6(1), pp. 262-76, 2012.

[7] R. K. Balan, K. X. Nguyen, \& L. Jiang, "Real-time trip information service for a large taxi fleet," Proceedings of the 9th International Conference on Mobile Systems, Applications, and Services, Bethesda, New York, NY: ACM, 2011.

[8] C. Narboneta, \& K. Teknomo, "OpenTripPlanner, OpenStreetMap, General Transit Feed Specification: Tools for disaster relief and recovery,"7th IEEEInternationalConferenceHumanoid, Nanotechnology, Information Technology. Puerto Princesa, New York, NY: ACM, 2016. 
[9] J. Scholtz, "Common industry format for usability test reports," Extended Abstracts on Human Factors in Computing Systems, The Hague, The Netherlands, New York, NY: ACM, 2000.

[10] K. Moumane, A. Idri, \& A. Abran, "Usability evaluation of mobile applications using ISO 9241 and ISO 25062 standards," SpringerPlus, 5(1), pp. 1-15, 2016.

[11] J. Sauro, \& E. Kindlund, "Making sense of usability metrics: Usability and six sigma," Proceedings of the 14th Annual Conference of the Usability Professionals Association. Montreal, Canada, Chicago, IL: UPA, 2005.

[12] J. Sauro, \& E. Kindlund, "A method to standardize usability metrics into a single score," Proceedings of the SIGCHI conference on Human factors in computing systems, Portland, Oregon, New York, NY: ACM, 2005.

[13] R. Alturki, \& V. Gay, "Usability testing of fitness mobile application: case study Aded Surat app.," International Journal of Computer Science \& Information Technology (IJCSIT), 9(5), pp. 107-127, 2017.

[14] R. Bringula, J. D. Depeno, J. J. Alpay, Z. M. Cruz, \& M. J. Altabirano, "Usability of "Fatchum": A mobile application recipe recommender system," Annual Conference on Research in Information Technology, Ronchester, New York, NY: ACM, 2017.

[15] P. Kortum, \& C. Z. Acemyan, "The relationship between user mousebased performance and subjective usability assessments," Proceedings of the Human Factors and Ergonomics Society Annual Meeting, 60(1), pp. 1174-1178, 2016.

[16] J. Sauro, \& J. R. Lewis, "Average task times in usability tests: what to report?," Proceedings of the SIGCHI Conference on Human Factors in Computing Systems, Atlanta, Georgia, New York, NY: ACM, 2010.

[17] J. Sauro \& J. S. Dumas, "Comparison of three one-question, post-task usability questionnaires," Proceedings of the SIGCHI conference on human factors in computing systems, Boston, New York, NY: ACM, 2009.

[18] D. Tedesco \& T. Tullis, "A comparison of methods for eliciting posttask subjective ratings in usability testing," Usability Professionals Association (UPA), Broomfield, Colorado, Taylor and Francis Online, 2006.

[19] J. Kiruthika, S. Khaddaj, D. Greenhill, \& J. Francik, "User Experience design in web applications," International Conference on Computational Science and Engineering, Paris, New York, NY: IEEE, 2016.

[20] P. Yan, \& J. Guo, "The research of web usability design," 2nd IEEE International Conference on Computer and Automation Engineering, Singapore, New York, NY: IEEE, 2010.

[21] E. Abana, M. Pacion, R. Sordilla R, D. Montaner, D. Agpaoa, R. M. Allam, "Rakebot: a robotic rake for mixing paddy in sun drying," Indonesian Journal of Electrical Engineering and Computer Science (IJEECS), 14(3), pp. 1165-1170, 2019.

[22] R. Shehzad, Z. Aslam, N. Ahmad, M. W. Iqbal, "Web usability and user trust on e-commerce websites in Pakistan," International Journal of Advanced Computer Science and Applications, 8(12), pp. 509-517, 2017.

[23] H. T. Basher, D. M. Gacus, R. P. Mingo, \& A. M. Ambe, "A usercentered evaluation of a university website," Journal of Industrial and Intelligent Information, 2(3), pp. 210-216, 2014.

[24] L. Wu, "Error Recovery in Human-Computer Interaction: A preliminary study in a database learning environment," Proceedings of the $2 \mathrm{nd} \mathrm{PhD}$ workshop on Information and knowledge management, Napa Valley, California, New York, NY: ACM, 2008.

[25] J. Sauro \& E. Kindlund, "How long should a task take? Identifying specification limits for task times in usability tests," Proceeding of the Human Computer Interaction International Conference, Las Vegas, CRC Press, 2005. 